$$
60 N=-820440-4
$$

FOLDING MODELS FOR ELASTIC AND INELASTIC SCATTERING*

G. R. Satchler

Oak Ridge National Laboratory Oak Ridge, TN 37830 , U.S.A.
CONF-620:AC- -4

DrS2 013 550

Talk to be presented at the Fifth Argentine Nuclear Physics Workshop, April 12-18, 1982, Chapadmalal, Argentina

\author{
Bu accebtance of this artiele ine

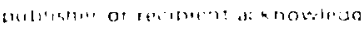 \\ the U.S Governmeing sront it \\ retalil a nonexclusite, rovalty tre: \\ license ir: and to anv copvrigh! \\ covering the article
}

\footnotetext{
* Research sponsored by the Division of Basic Energy Sciences, U.S. Department of Energy, under contract $W-7405$-eng-26 with the Union Carbide Corporation.
} 


\title{
FOLDING MODELS FOR ELASTIC AND INELASTIC SCATTERING*
}

\author{
G. R. Satchler \\ Oak Ridge National Laboratory \\ Oak Ridge, TN 37830 , U.S.A.
}

\section{Motivation}

We are accustomed to the use of many simple models in nuclear physics. in the present context, the most widely used models are the optical model potential (OMP) for elastic scattering, and its generalization to non-spherical shapes, the deformed optical model potential (DOMP) for inelastic scattering. These models are simple and phenomenolugical; their parameters are adjusted so as to reproduce empiricai data. Nonetheless, there are certain, not always we?1defired, constraints to be imposed. The potential shapes and their parameter values must be "reasonable" and should vary in a smooth and systematic way with the masses of the colliding nuclei and their energy. Without these constraints, the potentials tell us very little, and they will be essentially useless for other purposes, such as in DWBA calculations.

One way of satisfying these constraints, without going back to a much more fundamental theory, is through the use of folding models. Remember that the basic justification for using potentials of the Woods-Saxon shape for nucleonnucleus scattering, for example, is our knowledge that a nuclear density distribution is more-or-less constant in the nuclear interior with a diffuse surface. When this is fclded with a short-range nucleon-nucleon interaction, the result is a similar shape with a more diffuse surface. Folding procedures allow us to

\footnotetext{
${ }^{\star}$ Research sponsored by the Division of Basic Energy Sciences, U.S. Department of Energy, under contract W-7405-eng-26 with the Union Carbide Corporation.
} 
incorporate many aspects of nuclear structure (although the nuclear 'size' is one of the most important), as well as theoretical ideas about the effective interaction of two nucleons within nuclear matter. It also provides us with a means of linking information obtained from nuclear (hadronic) interactions with that from other sources, as well as correlating that from the use of different hadronic probes. For example, inelastic electron scattering measurements may provide a transition density $\rho_{t r}(\dot{r})$ for an excitation. Frequently this is recresented by a simple collective (deformed density) model when the transition is strong, whose shape may be of the form

$$
\rho_{t r}^{C}(\underline{r})=\beta_{L}^{C} R_{C} \frac{d \rho_{C}(r)}{d r} Y_{L}^{M}(\underline{r})
$$

where ${ }{ }_{C}(r)$ is the ground state charge density distribution, $R_{C}$ is its radius, and $B_{L}^{C}$ is an amplitude or 'charge deformation parameter' for the $2 L$-pole transition. The interaction for hadronic inelastic scattering is frequentiy represented in an analogous way (the DOMP) where the transition potential $U_{t r}(r)$ is taken to be

$$
U_{t r}(\underline{r})=\beta_{L}^{N} R_{N} \frac{d U(r)}{d r} Y_{L}^{M}(\underline{r})
$$

where $U(r)$ is the optical potential for elastic scattering of that particular hadron, $R_{N}$ is its radius, and $\beta_{L}^{N}$ is the corresponding 'nuclear' $2^{2}$-pole deformation parameter.

The use of these models (1) and (2) (and their extensioris) has led to much discussion as to how the $\beta^{N}$ and $\beta^{C}$ are related and, indeed, how the $\beta^{N}$ obtained with different probes are to be related. (One popular prejudicel is that the 'deformation lengths' should be the same, $\beta_{L}^{C} R_{C}=\beta_{L}^{N} R_{N}$.) Folding provides some insight into this problem $\mathrm{em}^{-4}$ and indeed may be used to construct the transition 
potential $U_{t r}(r)$ directly from the cransition density by folding with a suitable interaction. As usual, such extensions of a model also open up new uncertainties. For examp?e, the charge density (1) arises predominantly from proton excitations, while neutron and proton excitations contribute comparable anounts to the hadronic transition potential (2). However, we may turn this situation into an advantage and use it to give information about the neutrons.

Folding is still a model, and, at best, an approximation. It is a reasonable model for nucleon scattering. At low energies, it is closely related to the Brueckner-Hartree-Fock model for bound states. At high energies, it can be justified in terms of the impulse approximation. Extensions of the Bruecknertype theories provide support at intermediate energies. The situation is not so clear for composite projectiles, especially the scattering of two heavy ions. However, here the situation is helped because such systems usually exhitit strong absorption. Under such circumstances, the details of the interaction when the two nuclei overlap are largely irrelevant; all that matters is the interaction for peripheral collisions where again folding may be reasonable. Ultimately, the justification of the folding procedure mast be two-fold: (i) it must be seen to work in practice (i.e. to explain experimental data), and (ii) it should be justified from a more fundamental theory. This is the common fate of physical models.

\section{Further Theoretical Background}

\subsection{Coupled Equations and the Feshbach Projection Theory for the Optical Potential}

We have spoken so far as though the inter-nucleus potential was defined uniquely. This is not so; there are various ways in which we may reduce the compiicated $\left(A_{1}+A_{2}\right)$-body scattering problem to an effective two-body problem. 
Failure to remember this has sometimes led to confusion. A conventional optical model potential $U(R)$ for two nuclei $A_{1}, A_{2}$ is one which appears in a one-body Schrödinger equation

$$
\left[-\frac{\pi^{2}}{2 \mu_{\alpha}} \nabla^{2}+U(R)\right] x(R)=E \times(R),
$$

where $\mu_{\alpha}$ is the reduced mass of the pair, $R$ is the separation of their centers of mass, and $E$ is the CMS energy of relative motion. The solution $x(R)$ with the appropriate boundary conditions describes the elastic scattering of $A_{1}+A_{2}$. Usually $U(R)$ is assumed to be local (although its parameter values may vary with bombarding energy) and to have a smooth, simple functional form like that of the Woods-Saxon shape. Occasionally, some L-dependence is introduced, but only if one is forced to consider such a generalization ry some intractable data. The primary consideration is simplicity.

How do we justify the use of an equation like Eq. (3) which makes no reference to the many internal degrees of freedom and the corresponding nonelastic channels except insofar as they lead to absorption from the elastic channel and require $U$ to be complex? One standard way to make it plausible that such an equation may adequately describe elastic scattering is to use the project: on operator formalism of Feshbach. 5

Suppose that the complete Hamiltonian for the colliding pair is given by

$$
\mathrm{H}=\mathrm{H}_{1}+\mathrm{H}_{2}+\mathrm{T}+\mathrm{V}
$$

where $\mathrm{H}_{2}$ and $\mathrm{H}_{2}$ denote the internal Hamiltonians for the isolated nuclei, $T$ is the kinetic energy of relative motion in the center-of-mass system, and $V$ is the coupling interaction between the ions. At this point, we ignore any explicit reference to antisymmetrization between nucleons in different ions, although we assume that the wavefunctions for the internal states of the individual nuclei 
are antisymmetric. He denote a complete set of states for the internal Hamiltonians of nuclei $A_{1}$ and $A_{2}$ by $\psi_{i}$ and $\phi_{j}$ respectively, where

$$
\left(H_{1}-\varepsilon_{1 j}\right) \Psi_{i}=0, \quad\left(H_{2}-\varepsilon_{2 j}\right) \phi_{j}=0 \text {. }
$$

If the complete wavefunction is denoted by $\psi^{(+)}$, we note that measurements of elastic or inelastic scattering provide us with information about the projection of the complete wavefunction onto the channel subspace defined by the particular measurement. We denote these R-dependent projections (amplirudes) by

$$
x_{i j}(R) \equiv\left\langle\psi_{i} \phi_{j} \mid \psi^{(+)}\right\rangle,
$$

which means that we can express the tota? wavefunction as

$$
\psi_{\psi}^{(+)}=\sum_{i, j} x_{i j} \underset{\sim}{(R)} \psi_{i} \phi_{j} \cdot
$$

A set of coupled equations governing the $x_{i j}(\underset{\sim}{R})$ can be found by inserting the above expansion into the complete Schrödinger equation

$$
(H-E) \psi^{(+)}=0 \text {. }
$$

This yields

$$
\mid T+\left(\psi_{i} \phi_{j} \mid V i \psi_{i} \psi_{j}-E_{i j} j x_{i j}(\underset{\sim}{R})=\sum_{[k, \ell \neq i, j]}\left(\psi_{i} \dot{\phi}_{j} \mid V i \psi_{k} q_{\ell}\right) x_{k \ell}(R) .\right.
$$

where

$$
E_{1 j}=E-\varepsilon_{1 i-\varepsilon_{2 j}} \text { and }(i, j=0 \ldots \infty) \text {. }
$$

The rounded brackets denote integration over the internal coordinates of the two nuclei and serve to remind us that the matrix elements remain a function of $R_{0}$ Now Eqs. (9) represent an infinite set of coupled equations which, al though complete, are not very useful. Practical calculations require a severe 
truncation of these to a small set cf equations coupling a few states of particular interest; this is what is involved in the so-called 'coupled-channels' method. The use of an equation like Eq. (3) in fact involves truncation to just tinat one channel associated with the ground states, $i=j=0$. The price we pay for any such truncation is that the interaction $V$ should be replaced by an effective interaction. This becomes clear in Feshbach's rormalism ${ }^{5}$ in which all explicit couplings to channels other than the one of interest are transformed away and their effects incorporated into an effective interaction. For that channei in which both nuclei remain in their ground states this procedure results in an equivalent one-body Schrödinger equation for the $x_{00}(R)$,

$$
\left[T+U_{o p}-E\right] x_{o o}=0
$$

with the effective interaction or optical potential operator $U_{\text {op }}$ given by

$$
\begin{aligned}
U_{o p} & =\left(\psi_{O} \phi_{0}|V| \psi_{0} \phi_{0}\right)+\left(\psi_{D} \phi_{0}\left|V Q \frac{1}{E-H_{Q Q}+i \varepsilon} Q V\right| \psi_{O} \phi_{0}\right) \\
& =U_{F}+\Delta U .
\end{aligned}
$$

$U_{F}$ is defined as the first term : : Eq. (11)

$$
U_{F}=\left(\psi_{0} \phi_{0}|V| \psi_{0} \phi_{0}\right)
$$

and is called the folded potential for elastic scattering. (Note that this same folded potential and its generalization to excited states and off-diugonal couplings also appears in Eqs. (9).) $Q$ is the operator which projects off the ground states of the two nuclei, so that only excited states appear as intermediate states in $\Delta U$. By its construction, the exact elastic scattering amplitude may be obtained from $x_{00}$ as $R \rightarrow \infty$.

A simila: result is obtained when the truncation is to a model space which includes one or a few excited states as well as the ground states. $U_{o p}$ becomes a matrix in the model space and provides the effective interactions to be used 
in the corresponding truncated coupled-channels problem. In particutar, it yields (complex) corrections $\Delta U$ to the interchannel couplings in addition to the folded terms that already appear in the Eqs. (9).

The folded potential $U_{F}$ is real (provided that $V$ is real). The remaining term $\Delta U$, which we may refer to as a 'dynamic polarization potential', arises from coupling to all the other states, is much more difficult to calculate even approximately, and, in general, is complex, non-local, energy- and angularmomentum dependent. (In practice, because of the strong short-range repulsion in the bare nucleon-nucleon interaction, the $V$ itself will be an effective interaction or G-matrix which itself includes some 'polarization' corrections, primarily those associated with the short-range correlation between the interacting nucleons and hence high excitation energies. In principle, this leaves open the possibility of some double counting.)

In phenomenologica? approaches it is this object $U_{\text {op }}$ which is approximated by a local, romplex model potential $U(R)$ in Eq. (3). However, although this formalism provides us with the form of the optical model, it does not guarantee that any given simple representation of $U_{o p}$ will be adequate. This question may be explored both empirically (is a given model successful in fitting data?) and by studying the structure of $\Delta \mathrm{U}$.

\subsection{Other Approaches to the Nucleus-Nucleus Interaction Energy}

A particular point to be stressed is that with the potential $U_{\text {op }}$ (and by implication an equivalent model potential $U$ ) defined in this way, the solution $x(R)$ of Eq. (3) represents $x_{00}(R)$ of Eqs. (9) and (10) and describes the relative motion of the two nuclei while they both remain in their respective ground states. This may be a very small component of the total wavefunction in that region of space where the two nuclei overlap appreciably; in that case, the 
strong absorption into other channels manifests itself through $\chi_{00}(R)$ becoming very small for $R \leq R_{1}+R_{2}$.

This is to be contrasted with most of the potentials calculated microscopically for heavy-ion cullisions (see Refs. 6-10, for example). These calculations may use the energy-density approach, the Thomas-Fermi approximation, the liquid-drop model, the proximity theorem, etc., but they all attempt to follow to a greater or lesser degree the readjustments that the two nuclei must make as they begin to interact and overlap; distortion of the nuclear shapes, reaction to the Dauli principle, effects of the saturating nature of the nuclear forces, etc. Such an interaction energy function does not determine just the $x_{00}$ component of the wavefunction (7) but is to be used in a description of the motion of a wave packet which includes a wide range of excited states of the separated systems. It is not to be identified witr. $U_{\text {op }}$ of Eq. (11), and, in princip?e, it should not be used in an equation like Eq. (3).

It is possible that the equations for elastic scattering from these other approaches may be formally recast into the same form as the optical model Eq. (3) (see Pal, et al. ${ }^{11}$ for a recent discussion of this). The corresponaing effective optical potential $\tilde{U}$ appearing in such an equation will be different from ' op. It will contain not only the interaction energy function just referred to but also correction terms. For example, in one model situationlz there are corrections corresponding to changes in the kinetic energy due to changes in the inertial properties as the system begins to interact. The corresponding scattering solutions $\tilde{x}(\underset{\sim}{R})$ from such equations will differ from $x_{00}(\underline{R})$. Before approximations are made, we must have $\tilde{x}(\underline{R})+x_{00}(\underline{R})$ as ymptotically because the var ous theories describe the same elastic scattering, but these wavefunctions will differ in the interaction region at small $R$. 
The actual physical processes that are to be described in the two types of approaches are, of course, the same. In the Feshbach theory they manifest themselves in the dynamic polarization potential $\Delta U$; in some ways this formalism is deceptively simple, being most convenient to use when the effects of a few nonelastic channels are to be studied explicitly. The other approaches provide veficles for including, perhaps in intuitively more obvious ways, various bulk physical effects. Nonetheless, the 'potential' functions they generate should not simply be identified as optical potentials without bearing in mind the considerations al ready mentioned.

In practice, of course, approximations are made in both kinds of approach. trequently ar adiabatic aproximation is used when calculating an ion-ion interaction energy. This implies that the collision is slow enough for the two nuclei to readjust, both as they begin to interact and overlap and as they se: rate, so that they return to the elastic channel; there is no 'absorption' into non-elastic channels and the calculated interaction energy is real. The absorption into other channels that actually occurs must then arise from the correction terms mentioned above. In practice, these are usually replaced by a phenomenological absorptive potential. On the other hand, the folding model evaluates only the $U_{F}$ term of Eq. (11), identifying it with the real part of the potentidl and implicitly assuming that the polarization potential $\Delta U$ is predominantly imaginary and can be represented by a phenomenological imaginary potential. This corresponds to a sudden approximation in which excitations ('polarization') of the system tend not to de-excite back to the elastic channel, but result in absorption. 


\subsection{Folding Model Fotentials}

The idea of a folded potential has a long history. The electrostatic potential $U_{C}(r)$, due to a charge distribution $\rho_{0}(r)$, is given by a single folding,

$$
U_{C}(\underline{r})=\int \frac{\rho_{C}\left(r^{\prime}\right)}{\left|r-r^{\prime}\right|} d r^{\prime},
$$

while the electrostatic interaction between two charge distributions is given by a double folsing,

$$
U_{C}(R)=\iint \frac{{ }^{\rho} C 1\left(r_{1}\right) \rho_{C 2}\left(r_{2}\right)}{r_{12}} d{\underset{\sim}{1}}_{1} d r_{2}
$$

where $\underline{\sim}_{12}=\underline{R}+\underline{\sim}_{2}-\underline{r}_{1}$ (see Fig. 1). In the nuclear case, the coulomb interaction $1 / r_{12}$ is replaced by a nucleon-nucleon (effective) interaction $v_{12}$. The first term $U_{F}(R)$ in Eq. (11) was identified as a folded potential,

$$
U_{F}(R)=\iint \psi_{0}^{*}\left(\xi_{1}\right) \phi_{0}^{*}\left(\xi_{2}\right) \sum_{i j} v_{i j} \psi_{0}\left(\xi_{1}\right) \Phi_{0}\left(\xi_{2}\right) d \xi_{1} d \xi_{2}
$$

where nucleon $i$ is in nucleus 1 , nucleon $j$ is in nucleus 2, and $\xi_{1}$ and $\xi_{2}$ refer to the internal coordinates of these two nuclei, respectively. If $v_{i j}$ is a local interaction, $v_{i j}=v\left({\underset{\sim}{i j}}_{j}\right)$, then the potential (15) mav be reduced to the analogue of Eq. (14):

$$
\left.U_{F}(R)=\int \rho_{1}\left(\sigma_{2}\right) \rho_{2}\left(r_{2}\right) v ! r_{12}\right) d r_{1} d r_{2}
$$

where $\rho_{j}\left(\underline{r}_{j}\right)$ is the one-body density for the $i$ th nucleus, usually assumed to be spherically symmetric. This we call a double-folded potential. The properties and applications of this expression have been discussed in detail elsewhere (see Refs. 14-16), where many other references are also given). We just mention here that many results are more easily understood in momentum space by using fourier transforms. If we denote the transform of a function $f(\underline{r})$ by 


$$
\tilde{f}(\underline{q})=\int d \underline{\sim} \exp (\underline{i q} \cdot \underline{r}) f(\underline{r})
$$

then in momentum space Eq. (16) becomes

$$
\tilde{U}_{F}(q)=\tilde{v}(\underline{q}) \tilde{\rho}_{1}(\underline{q}) \tilde{\rho}_{2}(-q) \text {. }
$$

Then, for example, if the densities $\rho_{1}$ and $\rho_{2}$ are spherically symmetric,

$$
U_{F}(R)=\frac{1}{2 \pi^{2}} \int q^{2} d q j_{0}(q R) \tilde{v}(q) \tilde{\rho}_{1}(q) \tilde{\rho}_{2}(q) \text {. }
$$

When only small momentum transfers are involved in the scattering, it is helpful to expand the various factors in powers of $q$ in order to see which are their important characteristics. For example, if $f(r)$ is scalar in Eq. (17),

$$
\tilde{f}(q)=J_{0}\left[1-\frac{1}{6}\left\langle r^{2}\right\rangle q^{2}+\frac{1}{120}\left\langle r^{4}\right\rangle q^{4}+\ldots\right]
$$

where $J_{0}$ is the volume integral of $f(r)$ and $\left\langle r^{n}\right\rangle$ is its $n^{\text {th }}$ radial moment.

A simple generalization of the formula (16) is applicable to inelastic scattering.14-16 The transition potential for exciting one of the nuclei is obtained by replacing the corresponding ground-siate density by the appropriate transition density (for example, like the simple collective model one of Eq. (1)). The resulting folded transition potential is non-spherical (if $L \neq 0$ ). There is no reason to believe that its radial shape will be exactly like the radial derivative of the diagonal potential $U_{F}(R)$ of Eq. (16), as is assumed in the simple DOMP model of Eq. (2). indeed, it is known 15,15 that the folded shape depends upon the multipolarity, whereas the DOMP prescription (2) gives a shape that is independent of the multipole order.

We may break the double folding into two steps and rewrite Eq. (16):

$$
U_{F}(R)=\int d \underline{\sim}_{1} U_{2 N}\left(\underline{r}_{12}\right) \rho_{1}\left(\underline{\sim}_{1}\right),
$$


where

$$
U_{2 N}\left(\bar{r}_{12}\right)=\int d \underline{r}_{2} p_{2}\left(\underline{r}_{2}\right) v\left(\underline{r}_{12}\right)
$$

and $\bar{r}_{12}=\underline{r}_{12}-\underline{r}_{2}=\underset{\sim}{R}-\underline{\sim}_{1}$. Then $U_{2 N}$ is a folded potential for the interaction of nucleon 1 with nucleus 2 (analggous to Eq. (13)). Clearly Eq. (13) is the appropriate folding model for nucleon scattering from a nucleus. 13

\section{Single-Folded Potentials}

\subsection{Introduction}

In practice, the single-folded form (21) has often been invoked for the scaitering of composite nuclei, but with $U_{2 N}$ replaced by an empirical, phenomenological nucleon-nucleus $A_{2}$ optical potential, Usually this is what is meant by a single-folding model. Clearly the procedure is symmetrical in the two nuclei $A_{1}$ and $A_{2}$; one may equally well take an empirical nucleon-nucleus $A_{1}$ optical potentiai $U_{1 N}$ and fold it with the density distribution for nucleus $A_{2}$. Since the potentials $U_{1 N}$ and $U_{2 N}$ are empirical, there is no a priori guarantee that the two results will be identical.

The phenomenological nucleon-nucleus optical potential $U_{2 N}$ will be complex if it is obtained from analysis of nucleon-nucleus scattering. However, it should not be concluded that single-folding with the imaginary part wiil yield the correct imaginary potential for nucleus-nucleus scattering, even if use of the real part is successful. The imaginary part of $U_{2 N}$ accounts for absorption due to the excitation of one nucleus by a free nucleon; it does not account for excitation of the other nucleus in which the interacting nucleon resides, or effects due to the presence of the other nucleons in that nucleus. In the simplest case of the scattering of the loosely-bound deuteron, the absorption due 
to break-up of the deuteron is not included; the single-folding model gives an absorptive potential wich is much too weak.17 (Such break-up can also modify the effective real potential.18,19)

of course, if the potential $U_{2 N}$ (or its imaginary part) is not taken from empirical nucleon scattering data, but is adjusted to fit nucleus-nucleus scattering data, as in the alpha scattering case discussed below, then it is reasonable (though not necessarily correct!) to use the same adjusted interaction for, say, application to inelastic scattering.

The other ingredient of the single folding model is the density distribution for the nucleus into which the interaction is boing folded (or transition density, if an inelastic excitation is being considered). The tail of the folded potential, important for the scattering of strongly absorbed particles, is very sensitive to the extent of this density distribution, raising the possibility of learning something about the matter distribution provided some interaction potential can be shown to be valid. This is important because our other source of data on nuclear densities is electron scattering which primarily tells us about the proton distributions. Hadrons, on the other hand, interact as strongly with the nelitrons. Only for nuclei with $N=Z$ is it reasonabie to assume that neutron and proton distributions are the same (and even then there can be small differences because of the Coulomb forces). It is sometimes assumed that the distributions are in the ratio $N / Z$ when $N \neq Z$, or $\rho_{n}(r)=$ $(N / Z) \rho_{p}(r)$. Although reasonable, this assumption must be used with caution; it is believed, for example, that neutron distributions in the ground state extend to slightly larger radii than do the protons, and this can have quite large effects. 
The mean square radius (MSR) is a useful characteristic of the ground-state densities. Although the folded potential does depend to some extent upon the detailed surface shape of the density, it is largely determined by the MSR.15 In the case of $2^{L}$-pole excitations, this rôle is taken by the $r^{L+2}$ moment of the transition density. These results can be easily understood ${ }^{14-16}$ in momentum space, using the expansion (20).

A variety of representations of the ground-state density have been used. There is the staridard Fermi (= Woods-Saxon!) shape, or the convoluted form of Helm (itseif a fo?ding model) which has some analytic advartages. ${ }^{20}$ Independentparticle models have also been used, either from Hartree-Fock calculations or more phenomenological shell-model calculations.14,15 These provide a way of incorporating our prejudices about shell effects and about the behavior of the neutrons, for example.

Transition densities may be constructed in similar ways. One popular form is like that given by Eq. (1), except for the appearance of the matter, rather than the charge, distribution. Another is the Tassie or hydrodynamical model whose radial part differs from Eq. (1) by an additional factor of $r^{L-1}$; for $L>2$ and isoscalar excitations,

$$
\rho_{t r}(r)=\alpha_{L} r^{L-1} \frac{d \rho(r)}{d r} Y_{L}^{H_{1}}(\tilde{r})
$$

This has some appeal if the transition is strong (such as for a giant resonance), for it has been shown ${ }^{2}$ to be correct for a single transition that exhausts the classical energy-weighted sum rule (EWSR).

\subsection{Alpha Scattering}

Single fuiding has been applied with some success to alpha-nucleus elastic and inelastic scattering using either model $\mathrm{N}-\alpha$ potencials or ones derived from 
data on nucleon-alpha scattering. ${ }^{14}$ When we are concerned only with data for forward scattering angles (or small momentum transfers), the most relevant properties of the effective interaction are its strength (volume integral) and mean square radius (see Eq. (20)). In these circumstances, a simple form like a Gaussian may be used. A recent example $e^{22}$ adjusted the strength $-(V+i W)$ and range $\alpha$ of a Guassian $N-\alpha$ interaction to fit the forward angle $\left(\theta \leq 30^{\circ}\right)$ elastic scattering of $140-\mathrm{MeV}$ alphas. As had been found in earlier studies, ${ }^{14}$ there is a remarkable corsistency in the results (Fig. 2) with $V \approx 36 \mathrm{MeV}, \mathrm{W} \approx 23 \mathrm{MeV}$, and $\alpha \approx 1.94 \mathrm{fm}$.

This empirical interaction was then applied to inelastic data for the excitation of both low-excited states and giant-resonance states. The Tassie form (23) was adopted for the transition densities and the amplitudes $\alpha_{1}$ deduced by comparison with the data. Figure 3 shows an example for the lowest $3^{-}$state in $208 \mathrm{~Pb}$; the amplitude $\alpha_{3}$ required corresponds is $18 \%$ of the EWSR, which is exactly what one would deduce from the measured $B(E 3)$ value for this transition if it is assumed that the proton transition density is just ( $Z / A$ ) times the matter density. This illustrates how folding allows one to directly correlate information from different sources in a relatively unambiguous way. (The excellent agreement, of course, has to be somewhat fortuitous. Equation (23) is still a model; the proton and neutron contributions do not have exactly the same shape, and the proton part is not in exact agreement with the measured charge transition density. On the other hand, when a transition density of the form of Eq. (1) was used, the $B(E 3)$ value deduced from the $\left(\alpha, \alpha^{-}\right)$data was $50 \%$ larger than the measured one. We can conclude that the Tassie model (23) is more appropriate.) 


\subsection{Heavier Ions}

The single-folding approach to the optical potentials for heavy ions starts with some standard phenomenological nucleon optica? potential obtained from nucleon-nucleus scattering. It invariably overestimates the streryth of the real potential (near the strong absorption radius, where it matters most) by a factor of about two. ${ }^{23}$ Several reasons have been offered in explanation of this, 24 including the improper treatment of any density-dependence present in the underlying nucleon-nucleon effective interaction.

It has been suggested 25 that single folding of this kind may be adequate for estimating both the real and imacinary contributions to the optical potential from one or a few loosely-bound 'valence nucleons', even if it does fail for the bulk of the more tightly bound nucieons. In this approach, the nucieus is divided into 'core' and 'valence' oarts. The core contribution is taken to be the same as the phenomenological potential which describes scattering from the corresponding core nucleus, while the valence nucleons are treated 'microscopically' within the single-folding model. Successful applications have been made $^{25}$ to the elastic and inelastic scattering of ${ }^{17} 0$ anc ${ }^{18} 0$ where, of course, the core nucleus is taken to be ${ }^{16} 0$. The relatively weak binding of the valence nucleons results in their contribution to the interaction being of longer range than that due to the core nucleus. Thus, they may play a disproportionately large rôle in generating the potential at the large distances that are important for strongly absorbed scattering. In particular, they may have important effects on the Coulomb-nuclear interference pattern when the nucleus is excited. A recent example of the use of this model is shown in Fig. 4. The curves represent a preliminary analysis by Rhoades-Brown of Oak Ridge data ${ }^{26}$ for iho excitation of $120 \mathrm{MeV} 180$ to its first $2^{+}$state by scattering from $208 \mathrm{pb}$. The 
calculations are almost free of adjustable parameters. The complex core potential is taken from analysis of $130-\mathrm{MeV} 160+208 \mathrm{pb}$ elastic data. The interaction of the valence neutrons of 180 with the target is an empirical (complex) optical potential taken from analysis of $n+208 \mathrm{pb}$ scattering. The valence neutron wavefunctions result from shell-model calculations. ${ }^{25}$ These alone are not sufficierit to account for the $2^{+}$excitation; for example, being neutrons they would result in a vanishing $B(E 2)$ value unless they are assigned an effective charge. This implies polarization of the core protons. This core polarization is included ${ }^{25}$ by using the standard prescription (e.g., Eq. (21)) of deforming the core potential by an amount required to explain the observed $B(E 2)$ for this transition. The coupling is sufficiently strong that a first-order DWBA is not sufficient for a description of this excitation. Particularly imiportant is the re-orientation of the $2^{+}$state after its initial excitation (i.e. transitions between the various magnetic substates). The quadriapole component of this reorientation interaction is constructed in the same way, ${ }^{25}$ normalized to the observed electric quadrupole moment of the $2^{+}$state. In addition, it was found advantageous to include an hexadecapole interaction as well. Although there is no independent information on the electric hexadecapole moment of this $2^{+}$state, the values chosen are consistent with our expectations for nuclei in this region. The main effect of the additional reorientation due to this term is to decrease the cross section at the larger angles (see Fig. 4). (It is a cominon feature of this and other analyses of these data that such a term seems to be required. Consequently, we may obtain a measure of this hexadecapole interaction and, through folding, of the underlying mass moment.)

The agreement with the data is remarkable considering that no parameters were adjusted. The standard type of DOMP analysis has great difficulty in 
re,roducing accurately the Coulomb-nuclear interference minimum near $50^{\circ}$ without destroying the fit at other angles. Even the agreement shown in Fig. 4 probably could be improved by small parameter adjustments; this would be valid because all of the empirical quantities used to determine the input have appreciable experimental errors associated with them.

\section{The Double-Folding Model}

\subsection{The Ingredients}

The double-folding model has been reviewed in detail several times in the last few years (for example, see Refs. 15, 27, and 28). We on ly mention a few points here.

The discussion in Sec. 3.1 on the nuclear densities or transition densities applied equally here. The other ingredient in the formula (16) is the effective nucleon-nucleon interaction v. At one time (see Ref. 13 for example), it was popular to use for $v$ a simple pctential, such as a Gaussian, which fits lowenergy nucieon-nucleon scattering. However, this has been shown ${ }^{23}$ to overestimate the heavy-ion optical potential by :oughiy a factor of two. (It has also been shown ${ }^{29}$ to predict $\left(p, p^{-}\right)$cross sections which are too large.)

It is desirable to use ar effective interaction $v$ which is based upon a realistic nucleon-nucleon force, since one goal is to obtain a unified description of nucleon-nucleon, nucleon-nucleus and nucleus-nucleus scattering. As a consequence, our effective interaction is some kind of G-matrix. There is no guarantee of the existence of such a single, simple, effective interaction of this kind which may be used in a variety of physical circumstances. However, it is certainly worth seeing how far we can go with such a simple concept and to find what kind of corrections we may have to make in particular cases. 
A 'high-energy' approach to this problem was used by Dover and Vary, 16,28 In the high-energy limit (or impulse approximation), $v$ would become the (complex) t-matrix for free space nucleon-nucleon scattering. However, for the lowenergy ( $\lesssim 20 \mathrm{MeV}$ per nucleon) collisions that are encountered particularly in heavy-ion scattering, there are very large corrections to be made for the effects of the nuclear medium in which the two interacting nucleons are embedded (Pauli principle, off-shell propagation and Fermi motion of the nucleons). One 'low-energy' approach ${ }^{30}$ assumes that the effective interaction $v$ is similar tc the G-matrix for two nucleons bound near the Fermi surface. One consequence of this assumption is that $v$ is real, so that the imaginary interaction has to be treated phenomenologically. An exampie of this real interaction which has besil widely (and successfully) used 15,30 has become known as the MBY interaction. The part that is independent of spin and isospirl has the simple form

$$
V_{M 3 Y}(r)=\left[7999 \frac{e^{-4 r}}{4 r}-2134 \frac{e^{-2.5 r}}{2.5 r}\right]-262 \delta(r),
$$

where the ranges are in $i m$ and the strengths in MeV. The other spin, isospindependent terms have similar forms. The last, zero-range term in Eq. (24) is a pseudo-potential that represents the effects of knoci-on exchange between the incident and the struck nucleon. (This is believed to be the leading correction when the formula (16) is properly aritisymmetrized with respect to the interchange of nucleons between the two nuclei $A_{1}$ and $A_{2}$. The individual nuclear wavefunctions $\psi$ and $\phi$ are themselves assumed to be antisymmetric.)

Other work on nucleon-nucleus scattering 27,31 has taken into account that the projectile nucleon is in the continuum above the Fermi sea. This yields a complex G-matrix; however, it seems probable that at least the absorptive processes in the scattering of composite nuciei, especially heavy ions, are very 
different so that it may still be preferable to treat the imaginary potential phenomenologically. More recent work ${ }^{32}$ has recognized that, in the collision of two composite nuclei, both of the two interacting nucleons are embedded within two pieces of nuclear matter that are moving with respect to each other. This work provides both real and imaginary components of the interaction. The imaginary part provides the absorptive potential due to the bulk properties of the overlapping nuclei; it is supplemented by additional surface terms, calculated according to the $\Delta U$ term of Eq. (11), which arise from the finite extent of the two pieces of nuclear matter and correspond to the excitation of collective surface vibrations. ${ }^{3} 3$

It is worth remarking that replacing the bare nucleon-nucleon potential by an effective or G-matrix interaction is a process analogous to the derivation of the effective interaction $U_{\text {op }}$ in Sec. 2.1. The G-matrix transformation itself incorporates some polarization effects, namely the short-range correlations between pairs of nucleons and, in principle, allows us to use simpler nuclear wavefunctions such as those of the shell model. Then a full derivation of the operator $U_{o p}$ of Sec. 2.1 would go explicitly through this two-stage projection from the original many-body problem (see, for example, Ref. 34).

From its origin as an effective interaction or G-matrix, it is clear that, in general, this $v$ should be both energy-dependent and density-dependent. ${ }^{\dagger}$ For example, the volume integral of the effective interaction (including, implicit$7 y$, the knock-on exchange ${ }^{35}$ ) for a nucleon scattering from symmetric nuclear matter was found by Jeukenne, et al. ${ }^{31}$ to be represented approximately by

The MBY interaction (24) does not depend upon density or energy (except for a weak energy-dependence of the strength of the zero-range pseudo-potential). From its corstruction ${ }^{30}$ within a truncated space of oscillator functions, one can see that it represents a certain average over a range of kinetic energies and densities. 


$$
J_{0}(\rho, E) \approx F(E)\left(1-d \rho^{2 / 3}\right)
$$

with

$$
d=2.03 \mathrm{fm}^{2}, \quad F(E)=\left(903-7.67 E+0.22 E^{2}\right) \mathrm{MeV} \mathrm{fm}^{3}
$$

and the density $\rho$ in $\mathrm{fm}^{-3}$. ('Normal' nuclear matter has $\rho \approx 0.17 \mathrm{fm}^{3}$; then $1-\mathrm{d}^{2 / 3} \approx 0.38$, a large reduction from the value in free space.)

The usual way of handling the density dependence is to use the local density approximation. In this we assume that the effective interaction between two nucleons embedded in a nucleus at some position where the density has a certain value is given by the G-matrix for infinite nuclear matter with the same density value. This ignores the effects on $G$ of density gradients. Also, because the force has a finite range, two positions, $r_{1}$ and $\underline{r}_{2}$, are involved; frequently one uses the density at the center of gravicy, $\frac{1}{2}\left(\underline{r}_{1}+r_{2}\right)$. This kind of uncertainty is further aggravated ia heavy ion double folding because there are two densities, $\rho_{1}\left(\underline{r}_{1}\right)$ and $\rho_{2}\left(r_{2}\right)$. The simplest assumption is a sudden (or 'frozen density') approximation in which the two undisturbed densities are simply superposed. Then one uses $\rho=\rho_{1}+\rho_{2}$ in Eq. (25). This may be adequaite at hign energies (relative energy per nucleon high compared to the Fermi energy) where the Pauli principle has little effect and there is little time for the two nuclei to readjust as they collide. It seems less appropriate at lower energies; unfortunately, attempts to do better are quite complicated.32,33 Fortunately, the region of strong overlap is not experienced in most heavy-ion collisions because of the strong absorption, anc the frozen density approximation is more plausible for the low-density surface region. For a recent application to heavy-ion scattering, see Ref. 36. In the example of alpha scattering at 140 and $172 \mathrm{MeV}$ discussed below, one might argue that the energy per nucleon is 
moderately high and that the tight binding of the alpha discourages its polarization.

\subsection{Applications to Heavy-Ion Scattering}

The MBY interaction (24) has been used extensively to construct the real parts of optical potentials for the elastic scattering of heavy ions (see Refs. 15 and other references quated there). For example, analyses of some 156 sets of data for some 67 systems at erergies mostly between 5 and 15 MeV per nucleon were reported in Refs. 15. Except for $6,7 \mathrm{Li}$ and ${ }^{9} \mathrm{Be}$, these data were fitted by reiormalizing the real folded potential by a factor $\mathbb{N}$ which was found to be close to unity, $\bar{N}=1.06 \pm 0.11$. Although the imaginary potentials had to be chosen phenomenologically, good data do allor one to determine the strength required for the real part with little ambiguity.

Figure 5 shows another example of the use of the M3Y interaction. The experimental deformed potential contours were deduced ${ }^{37}$ from measurements on excitations of ${ }^{160} \mathrm{Gd}$ up to its $12^{+}$state by the inelastic scattering of ${ }^{40} \mathrm{Ar}$. The folded (non-spherical) potential was generated by using a non-spherical, quadrupole-deformed density for $160 \mathrm{Gd}$.

The exceptional cases of $6,7 \mathrm{Li}$ and ${ }^{9} \mathrm{Be}$ scattering require an opticai poten tial whose real part is only about half the strength predicted by the MY interaction. One feature that all three ions share is a dissociation energy much smaller than for other stable nuclei, so one is tempted to attribute the failure to the effects of break-up. This seems to have been confirmed, at least for ${ }^{6} \mathrm{Li}$, by some recent calculations. ${ }^{19}$ A similar overestmate of the real potential when the MBY interaction is used has been observed ${ }^{38}$ for the scattering of the light ions $d, t$, and ${ }^{3} \mathrm{He}$, which are also weakly bound. 


\subsection{Density Dependence and Alpha Scattering}

It was noted in Sec. 3.2 that a simple nucleon-alpha potential of Gaussian from was adequate, within the single-folding model, to reproduce the observed alpha scattering at forward angles. Use of the MBY interaction (24) in the double-folding model (plus a phenomenological imaginary potential) also provides agreement with data at 140 and $172 \mathrm{MeV}$ in the diffraction region at small angles, ${ }^{39}$ with only a few percent renormalization requircd for an optimum fit. This indicates that the model is providing the correct real potential for peripheral collisions.

It has long been known ${ }^{40}$ that observation of alpha scattering at energies high enough ( $\underset{\sim}{\sim} 100 \mathrm{MeV})$ and angles large enough to exhibit the 'nuclear rainbow' refractive effects can resolve the discrete ambiguities associated with the interior depth of the optical potential. Such data indicate that a real depth of $\sim 140 \mathrm{MeV}$ is required. However, the folded potential is $\sim 230 \mathrm{MeV}$ deep in the interior; consequently it will generate incorrect scattering at large angles. This is indicated in Fig. 6. Reducing the strength of the interaction (by $N \approx 0.5$ ) to give the correct large-angle scattering completely destroys the agreement at smaller angles because the potential in the surface is then too weak.

A natural way to rectify this situation is to introduce the expected density dependence, like that of $\mathrm{Eq}$. (25), which reduces the strength in the interior relative to that in the low-density surface region. Explicit calculations 39 confirmed that this was so, as shown by the example in Fig. 6. For these illustrative calculations, the MBY interaction (24) was simply multiplied by a density-dependent factor. This factorization is not strictly correct but makes the calculations easier. A factorized dependence on the densities, different from $(25 a)$, was adopted for computational convenience 1,42 


$$
v(r, \rho)=f(\rho) v_{M 3 Y}(r)
$$

with

$$
f(\rho)=C\left(1+a e^{-\beta \rho}\right), \quad \rho=\rho_{1}\left(r_{1}\right)+\rho_{2}\left(r_{2}\right) .
$$

The (energy-depc ndent) cristants in $f(p)$ were chosen to reproduce the magnitude and density dependence of the volume integral of the G-matrix of Jeukenne, et al. 31 at the appropriate energy per nucleon. An overall renormalization of the real folded potential by a factor of $N$ was allowed in fitting the 11 sets of alpha-scattering data. Quite consistently the optimum value was $N \approx 1.3$. With this, the factor $N f(\rho)$ is unity for a density of $\rho \approx 0.15 \mathrm{fm}^{-3}$, nearly equal to the 'ncrmal nuclear matter' density of $\rho_{0} \approx 0.17 \mathrm{fm}^{-3}$. This is somewhat la than had been expected, 15,41,42 but it is difficult to interpret this result precisely, in the light of the hybrid nature of the simple model interaction (26). We also note that the G-matrix of Ref. 31 is oniy given for $\rho \leqq \rho_{0}$, whereas larger values of $\rho=\rho_{1}+\rho_{2}$ are encountered when the alpha is within the target nucleus. Further, the use of $\rho=\rho_{1}+\rho_{2}$ may introduce too strong a dependence on density. The two densities are actually moving with respect to each other, this weakening the repulsive effect of the Pauli exclusion principle compared to that for the static superposition $\rho_{1}+\rho_{2}$. Further studies with a more sophisticated interaction would be of interes:. 


\section{References}

1. N. Austern and J. S. Blair, Ann. Phys. (Hew York) 33, 15 (1965).

2. A. M. Bernstein, Adv. Nucl. Phys. 3, 325 (1969).

3. R. S. Mackintosh and L. J. Tassie, Nucl. Phys. A222, 187 (1974); R. S. Mackintosh, Nucl. Phys. A266, 379 (1976).

4. A. M. Bernstein, V. R. Brown, and V. A. Madsen, Phys. Lett. 103B, 255 (1981); 106B, 259 (1.981).

5. H. Feshbach, Ann. Phys. (New York) 19, 287 (1967).

6. W. U. Schroder and J. R. Huizenga, Ann. Revs. Nuc1. Sci. 27, 465 (1977).

7. D. M. Brink, J. de Phys. C-5, 47 (1976).

8. Li. Mosel, in Heavy-Ion Collisions (ed. R. Bock: North-Holland).

9. U. Blocki, J. Randrup, W. J. Swiatecki, and C. F. Tsang, Ann. Phys. (New York) 105, 427 (1977).

10. H. J. Krappe, J. R. Nix, and A. Sierk, Phys. Rev. C 20, 992 (1979).

11. D. Pal, J. R. Rook, and A. M. Kobos, Nucl. Phys. A348, 45 (1980).

12. U. Mosel, Particles \& Nuclei 3, 297 (1972).

13. G. W. Greenlees, et a1., Phys. Rev. 171, 1115 (1968); C 1, 1145 (1970); C 2, 1063 (1970).

14. R. C. Barrett and D. F. Jackson, Nuclear Sizes and Structure (Clarendon Press, 0xford, 1977).

15. G. R. Satchler and W. G. Love, Phys. Reperts 55C, 183 (1979); G. R. Satchler, Nuci. Phys. A329, 323 (1979).

16. F. Petroyich, Nucl. Phys. A25î, 143 (1975); P. J. Moffa, C. B. Dover, arıd J. P. Vary, Phys. Rev. C 16, 1857 (1977).

17. F. G. Perey and G. R. Satchler, Nucl. Phys. A97, 515 (1967). 18. P. W. Keaton and D. D. Armstrons, Phys. Rev. C 8, 1692 (1973). 
19. I. J. Thompson and M. A. Nagarajan, Phys. Lett. 106B, 163 (1981).

20. H. J. Krappe, Ann. Phys. (New York) 99, 142 (1976).

21. E. I. Kao and S. Fallieros, Phys. Rev. Lett. 25, 827 (1970); H. Ui and T. Tsukamoto, Prog. Theor. Phys. 51, 1377 (1974).

22. F. E. Bertrand, G. R. Satchlei, D. J. Horen, J. R. Wu, A. D. Bacher, G. T. Emery, W. P. Jones, and D. W. Miller, Phys. Rev. C 22, 1832 (1980).

23. G. R. Satchler, Phys. Lett. 59B, 121 (1975).

24. L. D. Rickertsen and G. R. Satchler, Phys. Lett. 666, 9 (1977); F. Detrovich, D. Stanley, and J. J. Bevelacqua, Phys. Lett. 71 B, 259 (1977); W. G. Love, Phys. Lett. 72B, 4 (1977).

25. S. Landowne, R. Schlicher, and H. H. Wolter, Nucl. Phys. A373, 141 (1982). 26. E. E. Gross, J. R. Beene, K. A. Erb, M. P. Fewell, D. Shapira, M. J. Rhoades-Brown, G. R. Satchler, and C. E. Thorn, Bull. Am. Phys. Soc., Washington meeting, April, 1982, and to be published.

27. H. V. von Geramb, Microscopic Optical Potentials (Springer-Verlag, Berlin, 1979).

28. C. B. Dover and J. P. Vary, in Symposium on Classical and Quantum Mechanical Aspects of Heavy-Ion Collisions, Heidelberg, 1974 (SpringerVerlag, Berlin, 1975).

29. E. C. Halbert and G. R. Satchler, Nucl. Phys. A233, 265 (1974); G. R. Satchler, Zeit. f. Phys. 260, 209 (1973).

30. G. Bertsch, J. Borysowicz, H. McManus, and W. G. Love, Nucl. Phys. A284, 399 (1977); G. R. Satchler and W. G. Love, Phys. Lett. 65B 415 (1976).

31. J. P. Jeukenne, A. Lejeune, and C. Mahaux, Phys. Rev. C 16, 80 (1977); F. A. Brieva and J. R. Rook, Nucl. Phys. A29l, 317 (1977); F. A. Brieva, H. V. von Geramb, and J. R. Rook, Phys. Lett. 79B, 177 (1978). 
32. A. Faessier, T. Izumoto, S. Krewald, and R. Sartor, Nucl. Phys. A359, 509 (1981).

33. S. B. Khadkikar, L. Rikus, A. Faessler, and R. Sartor, Nucl. Phys. A369, 495 (1981).

34. V. A. Madsen, in Nuclear Spectroscopy and Reactions, Part D (ed. J. Cerny, Academic Press, New York, 1975).

35. J. P. Jeukenne and C. Mahaux, Zeit. f. Phys. A302, 233 (1981).

36. F. J. Vinas, M. Lozano, and G. Madurga, Phys. Rev. C 23, 780 (1981).

37. R. E. Neese, M. W. Guidry, R. J. Donange10, and J. 0. Rasmussen, Phys. Lett. 85B, 201 (1979).

38. J. Cook and R. J. Griffiths, Nucl. Phys. A366, 27 (1981); J. Cook, to be published.

39. A. M. Kobos, B. A. Brown, P. E. Hodgson, G. R. Satchler, and A. Budzanowski, Nucl. Phys. A (in press).

40. D. A. Goldberg, S. M. Smith, and G. F. Burdzik, Phys. Rev. C 10, 1362 (1974).

41. W. G. Love, Phys. Lett. 72B, 4 (1977).

42. F. Petrovich, D. Stanley, and J. J. Bevelacqua, Phys. Lett, 71 B, 259 (1977). 


\section{Figure Captions}

Fig. 1. Coordinates used in folding calculations.

Fig. 2. Variation of $x^{2}$ with the range of the Gaussian nucleon-alpha interaction used in a single-folding model when real and imaginary strengths are optimized to fit elastic data at forward angles. 22

Fig. 3. Comparison of single-folding moiel predictions, 22 using $18 \%$ of the energy-weighted sum rule with measured cross sections for the $3^{-}$state in $208 \mathrm{pb}$.

Fig. 4. Coupled-channels predictions using the hybrid folding model of Landowne, et al. ${ }^{25}$ for exciting the first $2^{+}$state of 180 when scattering at $120 \mathrm{MeV}$ from $208 \mathrm{pb}$, compared to measured cross sections. 26 The solid curve includes reorientation due to a hexadecapole moment of the $2^{+}$state, the dashed curve does not. (Both include reorientation due to the quadrupole moment.)

Fig. 5. Non-spherical optical potential contours ${ }^{37}$ for ${ }^{40} \mathrm{Ar}+160 \mathrm{Gd}$, referred to the body-fixed axes for $160 \mathrm{Gd}$, compared to folding-model calculations using the MBY interaction.

Fig. 6. Comparison of measured elastic scattering of $\alpha+58 \mathrm{Ni}$ with doublefolding calculations using either the MBY interaction (dashed curve) or the same interaction modified by a density-dependent strength (full curve). 

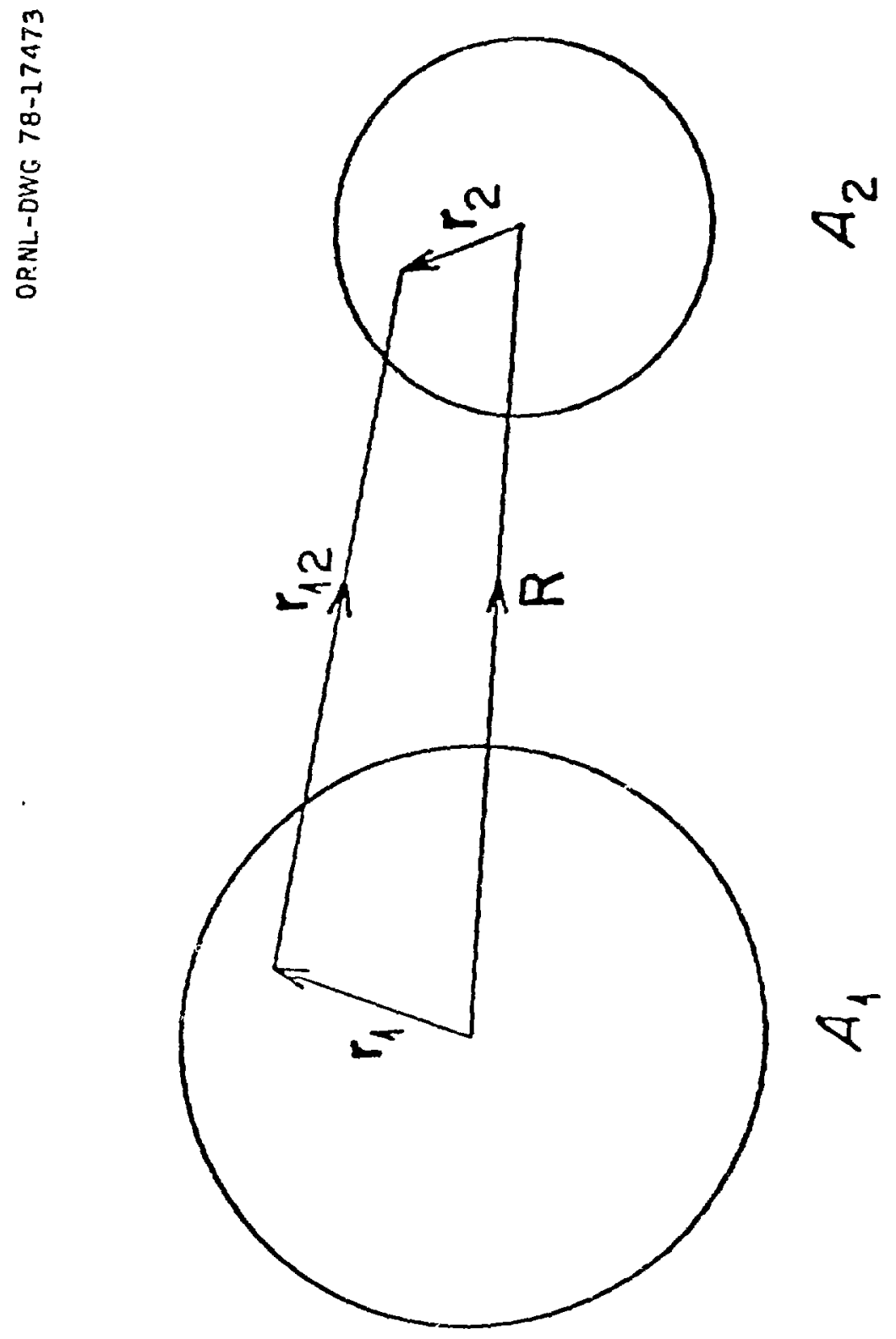

Fig. 1 


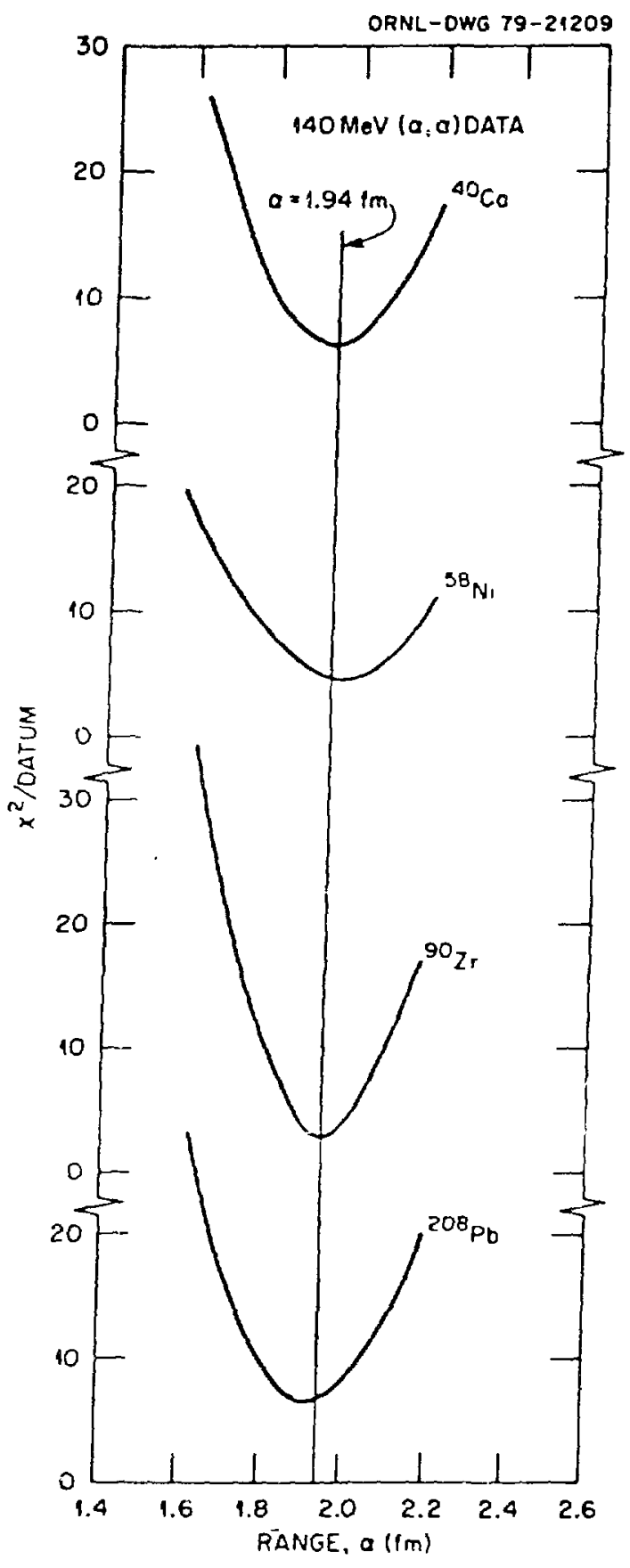

Fig. ? 


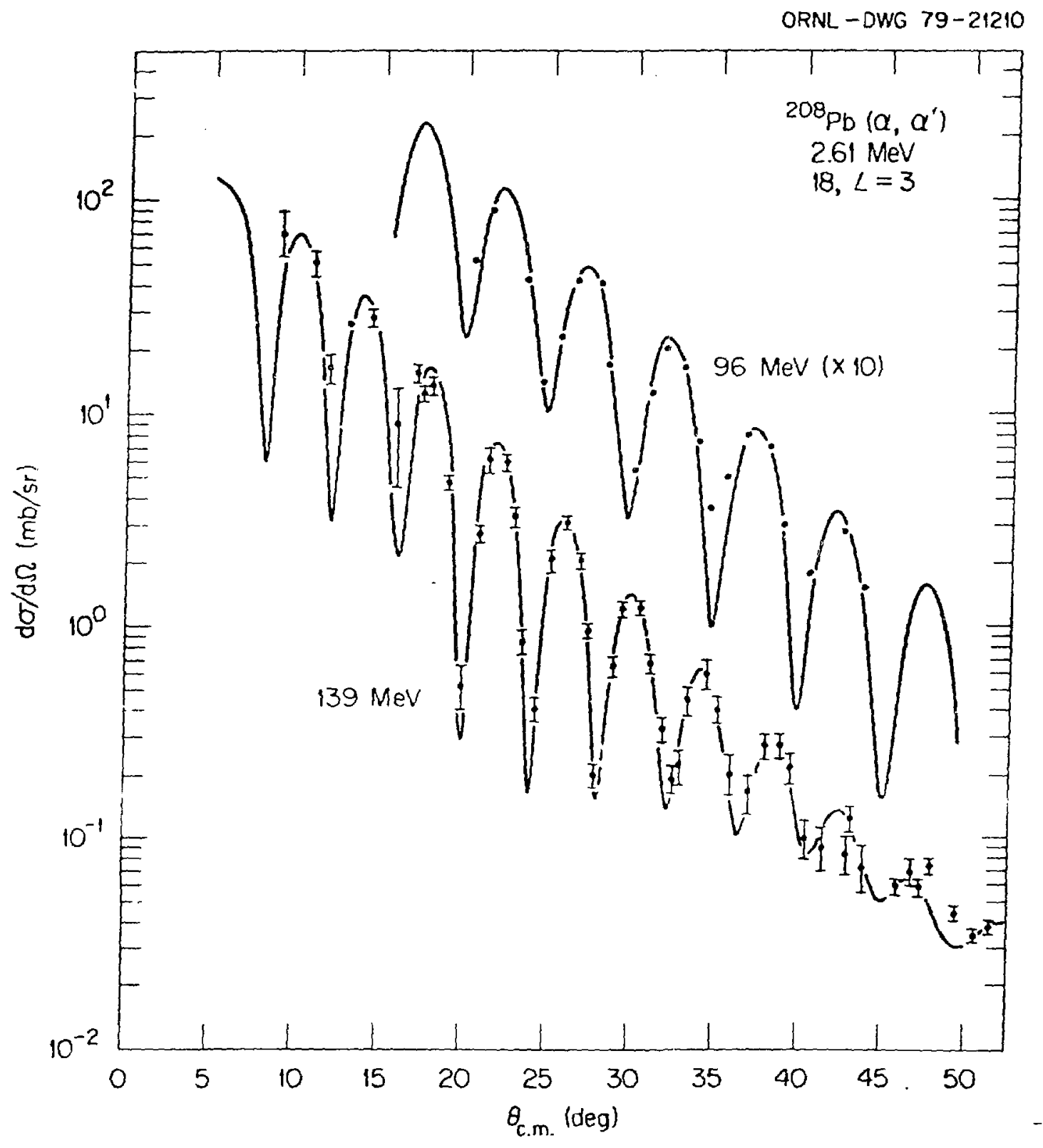

Fig. 3 


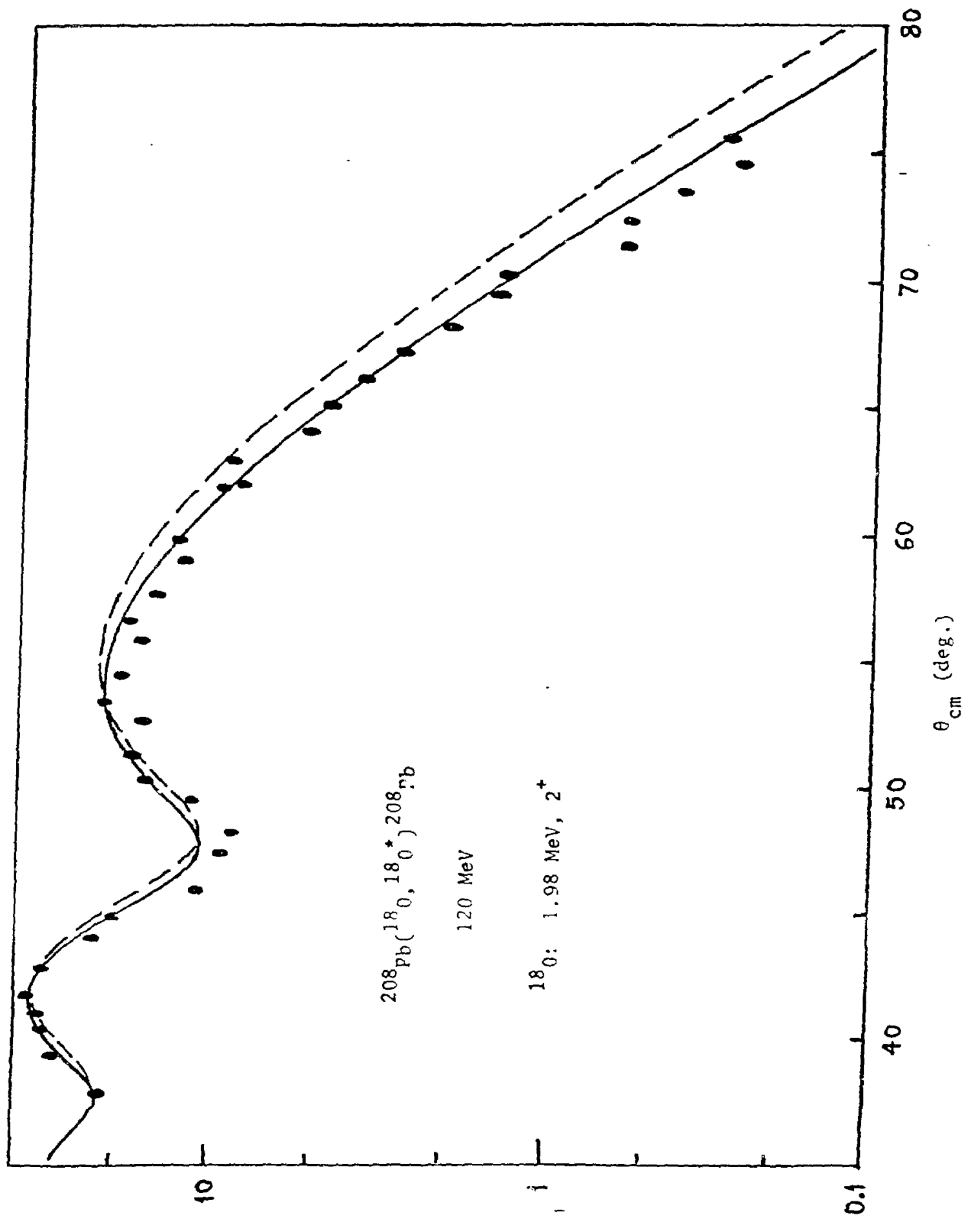

(Is/qu) $2 p / O p$

Fig. 4 


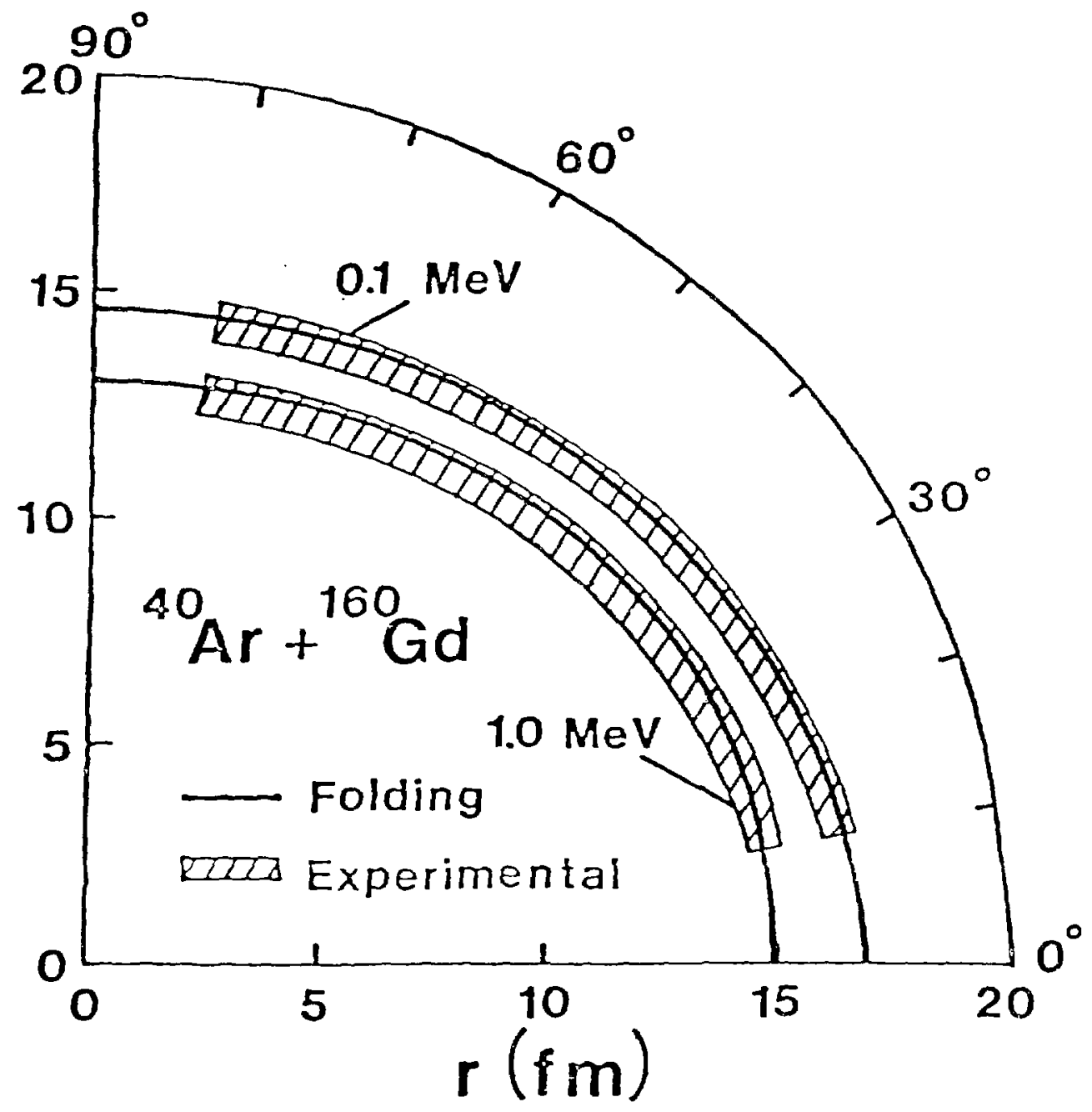

Fig. 5 


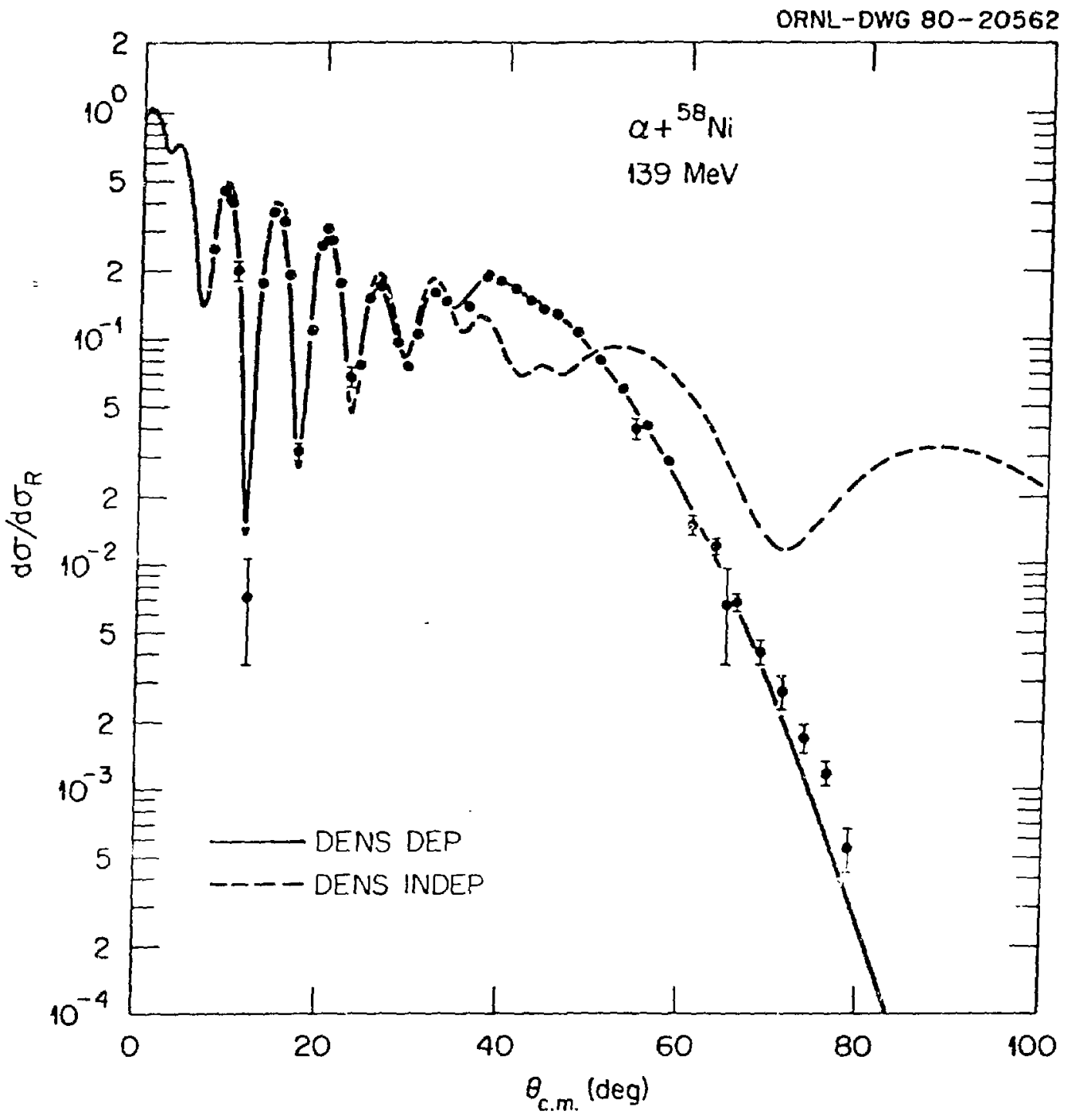

Fig. 6 